\title{
Quantitative proteomic analysis of S-nitrosated proteins in diabetic mouse liver with ICAT switch method
}

\author{
Xu Zhang ${ }^{1,2 *}$, Bo Huang ${ }^{1,2^{*}}$, Xixi Zhou ${ }^{1,2}$, Chang Chen ${ }^{1 凶}$ \\ ${ }^{1}$ National Laboratory of Biomacromolecules, Institute of Biophysics, Chinese Academy of Sciences, Beijing 100101, China \\ ${ }^{2}$ Graduate School of the Chinese Academy of Sciences, Beijing 100049, China \\ \ Correspondence: changchen@moon.ibp.ac.cn \\ Received June 9, 2010 Accepted June 22, 2010
}

\begin{abstract}
In this study we developed a quantitative proteomic method named ICAT switch by introducing isotope-coded affinity tag (ICAT) reagents into the biotin-switch method, and used it to investigate $S$-nitrosation in the liver of normal control C57BL/6J mice and type 2 diabetic KK-Ay mice. We got fifty-eight $S$-nitrosated peptides with quantitative information in our research, among which thirty-seven had changed S-nitrosation levels in diabetic mouse liver. The $S$-nitrosated peptides belonged to fortyeight proteins (twenty-eight were new S-nitrosated proteins), some of which were new targets of S-nitrosation and known to be related with diabetes. S-nitrosation patterns were different between diabetic and normal mice. Gene ontology enrichment results suggested that $S$-nitrosated proteins are more abundant in amino acid metabolic processes. The network constructed for $S$ nitrosated proteins by text-mining technology provided clues about the relationship between $S$-nitrosation and type 2 diabetes. Our work provides a new approach for quantifying $S$-nitrosated proteins and suggests that the integrative functions of $S$-nitrosation may take part in pathophysiological processes of type 2 diabetes.
\end{abstract}

KEYWORDS ICAT switch, mass spectrometry, quantitative, S-nitrosation, type 2 diabetes

\section{INTRODUCTION}

It is well established that nitric oxide (NO) can regulate a diverse array of signal transduction pathways which play important roles in both physiologic and pathological processes, partly acting through modification of cysteine residues of proteins (S-nitrosation) (Hess et al., 2005). Methods such as biotin-switch and SNOSID have been developed for studying S-nitrosation (Jaffrey and Snyder, 2001; Hao et al., 2006). However, these approaches will not be powerful enough for those researches concerning quantitative analysis of $S$-nitrosation. As known, the biotin switch method includes three key steps: blocking free cysteines, specific reduction of nitrosated cysteines (Snitrosothiol, SNO) by ascorbate, and biotinylation of released free cysteines by biotinylation reagents such as biotin-HPDP. Then the biotinylated proteins or peptides can be purified by streptavidin-agarose for further analysis. Isotope-coded affinity tag (ICAT) reagents, reactive to free thiols, have been used extensively in quantitative proteomics (Gygi et al., 1999). Here, we replaced the biotin-HPDP in the original biotin-switch method with ICAT reagents following with mass spectrometry detection, allowing quantification of $S$ nitrosated proteins in samples at the proteomic level. This method was named ICAT switch.

Type 2 diabetes mellitus (T2DM) is now a major threat to human health (Zimmet, 2000). Although the pathology of the development of T2DM is complicated, chronic inflammation induced by obesity is thought to play a key role (Wellen and Hotamisligil, 2005). The expression of inducible NO synthase (iNOS) increases under inflammation conditions (Xie et al., 1994), and targeted disruption of iNOS protects against obesity-linked insulin resistance in muscle tissue (Perreault and Marette, 2001). Endogenous NO produced abnormally by iNOS has a variety of effects on some processes in the development of diabetes (Perreault and Marette, 2001; Sugita

"These authors contributed equally to the work. 
et al., 2002; Fujimoto et al., 2005) and its complications (Ceriello et al., 2002), and alterations in the amount of Snitrosated proteins such as insulin receptors, insulin receptor substrate 1, and Akt (Carvalho-Filho et al., 2005; Yasukawa et al., 2005; Wadham et al., 2007) are reported to be involved in these diseases. Liver is an important organ for many metabolic processes, such as gluconeogenesis, glycogenesis, fatty acid and triglyceride metabolism, many of which are abnormal in diabetes. Research on S-nitrosated proteins in liver at the proteomic level will help to uncover the role of $S$ nitrosation in diabetes.

In the present study we developed the ICAT switch method to identify and quantify $S$-nitrosated proteins in the liver of control C57BL/6J mice and diabetic model KK-Ay mice. The relationship between $S$-nitrosated proteins and diabetes was subsequently analyzed using bioinformatics.

\section{RESULTS}

\section{Construction and validation of ICAT switch method}

We labeled the S-nitrosated thiols in C57BL/6J samples with light ICAT reagents and the S-nitrosated thiols in KK-Ay samples with heavy ICAT reagents. The peptides containing both quantitative and SNO site information were then identified by mass spectrometry. The flow chart of ICAT switch is shown schematically in Fig. 1A (ICAT structure was shown in Supplemental Fig. 1), in which ICAT reagents were used to replace biotin-HPDP to label the S-nitrosated Cys residues. Taking one result as an example, the peaks of peptide AGCQVVPSDMMDGR labeled with ICAT reagents can be seen in precursor MS spectrum as shown in Fig. 1B. The difference in $\mathrm{m} / \mathrm{z}$ units between light and heavy ICAT labeled peptides is $9 \mathrm{Da}$ for univalent ions, $4.5 \mathrm{Da}$ for divalent ions, and $3 \mathrm{Da}$ for trivalent ions. This spectrum indicated that ICAT reagent could be used to identify $S$-nitrosation with mass spectrometry.

To test the accuracy of ICAT switch, we applied this method in quantifying $S$-nitrosation induced by $S$-nitrosoglutathione (GSNO) in vitro, whose ratio could be controlled. The liver lysates of normal mice were treated with $500 \mu \mathrm{M}$ GSNO for protein S-nitrosation followed by ICAT switch. Half was biotinylated by $\mathrm{L}$ (light) and another half by $\mathrm{H}$ (heavy) ICAT reagent. Since the levels of $S$-nitrosation in both halves of the

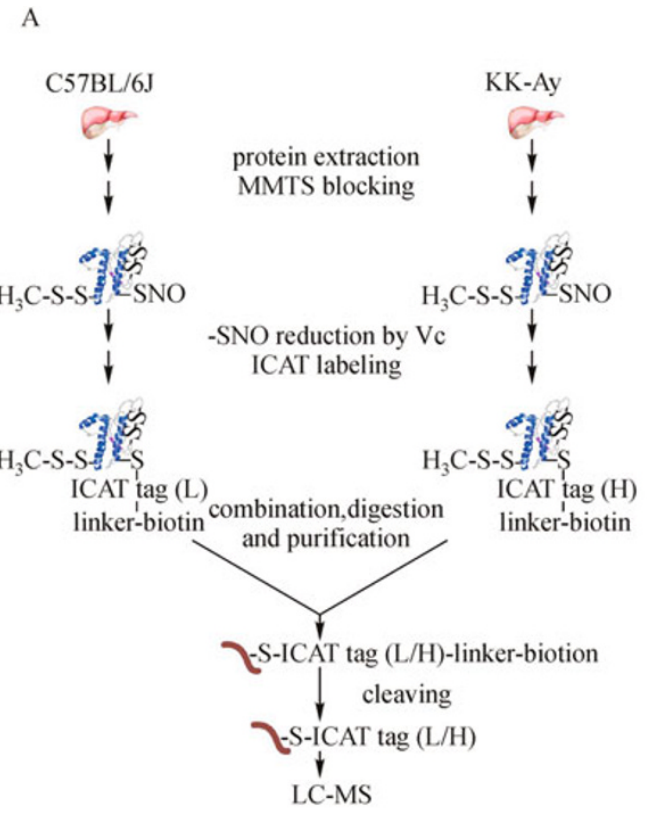

B

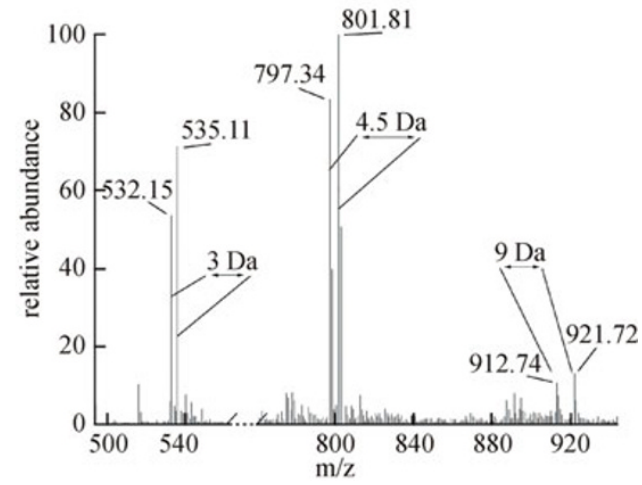

C

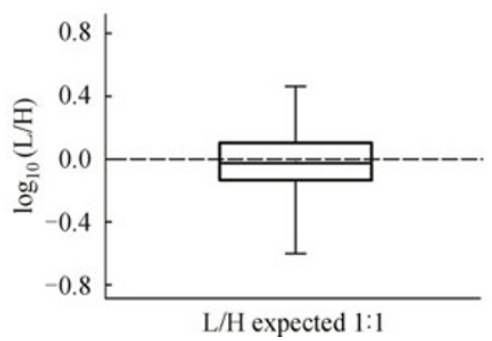

Figure 1. Construction and validation of the ICAT switch method. (A) Schematic overview of ICAT switch. Proteins were extracted from mouse liver and added to a blocking buffer, in which the free Cys-thiols were methylthiolated by MMTS. S-NO bonds in proteins from C57/BL6 and KK-Ay mouse liver were then separately reduced by ascorbate and labeled with ICAT light/heavy reagents. Samples from C57/BL6 and KK-Ay mouse liver were then combined and digested by trypsin at $37^{\circ} \mathrm{C}$ overnight. Peptides labeled with ICAT reagents were purified by monomeric avidin agarose, and then acid-cleaved by cleaving reagents. Finally, peptides were analyzed by LC-MS/MS. (B) Representative mass spectrum of the ICAT labeled peptides in full scan with three kinds of mass intervals. The difference of mass values between the isotopic peptides was $9 \mathrm{Da}$ for univalent ions, $4.5 \mathrm{Da}$ for divalent ions, and $3 \mathrm{Da}$ for trivalent ions. (C) Box plot of light/heavy ratios of $S$-nitrosated peptides is shown. The middle line in the box indicates the median of light/heavy ratios in this group of data. The box indicates $50 \%$ members of the group. 
lysates were the same, the expected value of the ratios was $1.0(\mathrm{~L} / \mathrm{H}=1: 1) .127 \mathrm{~S}$-nitrosated peptides were identified with an average ratio 0.94 and the distribution was shown in Fig. 1C. These results guaranteed the accuracy of our method used afterward.

\section{S-nitrosated peptides and proteins identified in C57BL/6J and KK-Ay diabetic mouse liver}

We analyzed the different $S$-nitrosation patterns between normal and diabetic mouse liver by ICAT switch. The probability above 0.8 for every peptide acquired by Peptideprophet was set as the criteria for peptide acceptance. Filtration of $S$-nitrosated peptide was shown in Fig. 2A. Employing a threshold of Probability above 0.8 , the percent of false $S$-nitrosated peptides identified in this experiment is below $3.6 \%$. In this state, the fraction of all correct assignments passing the minimal probability thresholds (MPT) filter is nearly $75 \%$ (sensitivity). The accepted peptides contained Cys were quantified afterward by ASAPRatio, which was part of the TPP software package. The peptide AGCQVVPSDMMDGR in aminolevulinate delta dehydratase (gi 34328485) was taken as an example, in which heavy ICAT reagents were labeled on the cysteine (Fig. 2B). There was a shift of 236 (the mass of the heavy ICAT tag) in the $\mathrm{m} / \mathrm{z}$ from the $b_{3}$ to the $b_{13}$ ion and an ICAT diagnostic peak at $\mathrm{m} / \mathrm{z}$ 294.9 (cl). Fig. 2C shows quantification of the peptide AGCQVVPSDMMDGR by ASAPRatio. The ratio of signals from light (C57BL/6J) versus heavy (KK-Ay) ICAT labeled peptides was determined to be $0.46 \pm 0.03$, meaning that the amount of $S$-nitrosated peptides in KK-Ay increased to about 2.2 fold $(1 / 0.46)$ that of C57BL/6J. S-nitrosated peptides whose amount is changed in diabetic mouse liver $(\mathrm{L} / \mathrm{H}<0.7$ or $\mathrm{L} / \mathrm{H}>1.3)$ were listed in Table 1 , and the others $(0.7 \leqslant L / H \leqslant 1.3)$ were shown in Supplemental Table 1. After checking the MS/MS spectra and the SIC (single ion chromatogram) manually by ASAPRatio, 58 peptides were considered as $S$-nitrosated peptides containing quantitative information. The distribution of the peptides ratios is shown in Fig. 3A. Of the 58 peptides identified, 24 peptides whose L/H ratio was less than 0.7 were considered as $S$-nitrosation increased in KK-Ay mouse liver, 13 peptides whose L/H ratio was more than 1.3 were considered as $S$-nitrosation decreased, and 21 peptides whose $\mathrm{L} / \mathrm{H}$ ratio was between 0.7 and 1.3 were considered to show no change in $S$ nitrosation status, because changes below $\pm 30 \%$ were considered as not significantly different in accordance with the ICAT kit instructions. An L/H ratio of 0 meant that the $S$ nitrosated peptide was only present in KK-Ay mouse liver, and an $\mathrm{L} / \mathrm{H}$ ratio of 999 meant that the $S$-nitrosated peptide was only present in C57BL/6J mouse liver.

\section{Bioinformatic analysis of S-nitrosated proteins}

To identify which biological processes were more likely to be regulated by quantitative changes in S-nitrosated proteins, we performed $\mathrm{GO}$ enrichment of these proteins using the BiNGO plugin in the Cytoscape platform. Thirty proteins with altered levels of S-nitrosation in KK-Ay mouse liver were analyzed by $\mathrm{GO}$ enrichment tool BiNGO. The enriched GO terms were visualized using a DAG (directed acyclic graph) graphical representation (Fig. 3B). The "amino acid metabolic processes" had a low $p$-value, which meant this process had higher probability to be regulated by $S$-nitrosation proteins. Other biologic processes enriched are listed in Supplemental Table 2.

Since S-nitrosated proteins may affect signaling pathways indirectly through their impact on other proteins (Benhar and Stamler, 2005; Qu et al., 2007), we constructed a protein network containing $14 \mathrm{~S}$-nitrosated proteins identified in KKAy mouse liver and other related proteins by virtue of textmining technology to find out the latent functions of the $S$ nitrosated proteins (Fig. 4A). Green, white and red nodes stand for $S$-nitrosated proteins, and the gradient from green to red reflects the $S$-nitrosation change from increasing to decreasing. Grey nodes stand for related proteins to $S$ nitrosated targets. The edge between two nodes stands for the relationship between them. For example, Fig. 4B shows a subnet composed of an S-nitrosated protein and its related proteins. In this subnet, the green color of the node for serine/ threonine kinase receptor-associated protein (STRAP), which was identified as an $S$-nitrosated protein in our research, means that the amount of $S$-nitrosated STRAP increased in $\mathrm{KK}-\mathrm{Ay}$ mouse liver. Other gray nodes stand for proteins related with STRAP. Of the related proteins, the edge between Cdkn1a (an alias of p21) and STRAP shows that stable expression of STRAP induces downregulation of the cyclin-dependent kinase inhibitor p21 (Halder et al., 2006); the edge between trp53 (an alias of p53) and STRAP shows that STRAP activates p53 (Jung et al., 2007).

\section{DISCUSSION}

Here we developed the ICAT switch method for quantitative proteomic research on $S$-nitrosation in diabetic mouse liver. By virtue of the ICAT switch in quantification, we solved the problem of false negative identification of $S$-nitrosation targets. In our study there are 20 peptides with $0<\mathrm{L} / \mathrm{H}<0.7$ and seven peptides with $1.3<\mathrm{L} / \mathrm{H}<999$, which were identified as new $S$-nitrosation targets and their quantitative variation was determined. However, using former methods lacking quantitative information, these proteins would have been eliminated from the $S$-nitrosated protein target list since they were presented in both control C57/BL6 and the $\mathrm{KK}-\mathrm{Ay}$ mouse liver, therefore, leading to false negative identification of S-nitrosation targets. Some S-nitrosated targets reported in other studies, such as transaldolase (Lefièvre et al., 2007), glutathione S-transferase (Sies et al., 1998; Lindermayr et al., 2005; Hao et al., 2006; Lefièvre et al., 2007), D-dopachrome tautomerase (Han and Chen, 2008), 
A

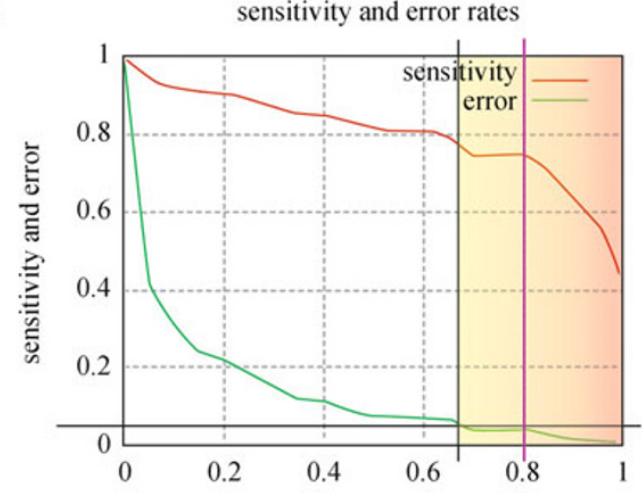

minimal probability threshold (MPT) To accept

B

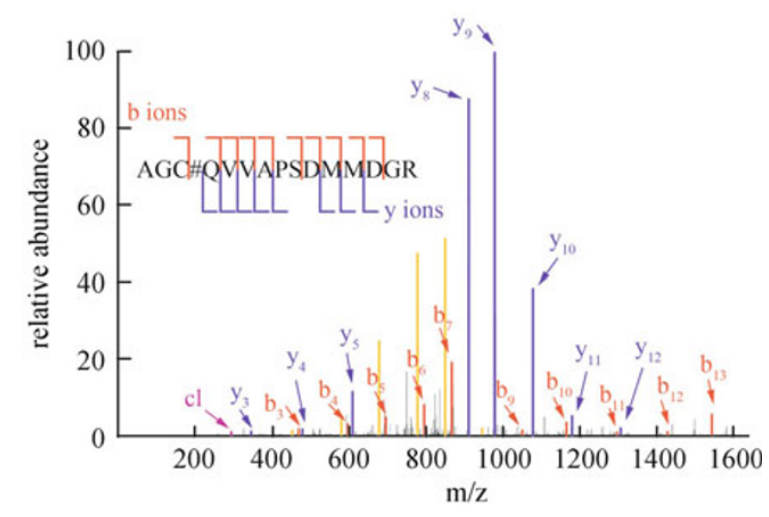

C
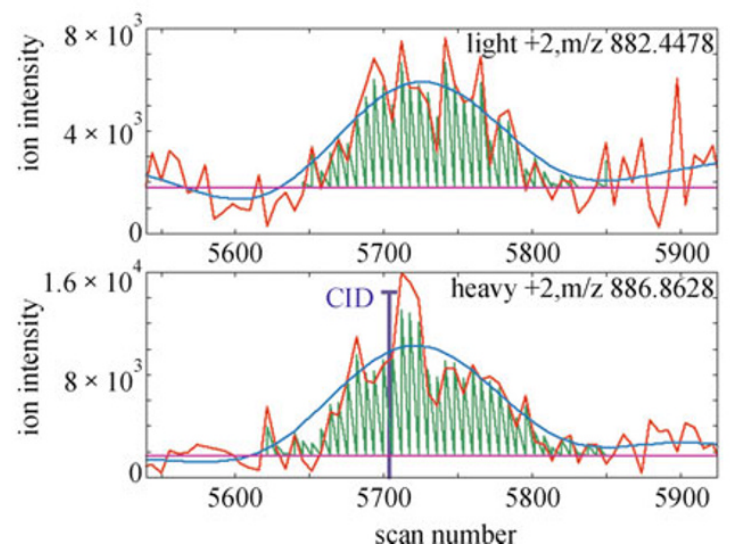

Figure 2. Filtering, identification and quantification of S-nitrosated peptides. (A) Filtration of S-nitrosated peptide. The MPT for false positive (error) rate below 0.05 is 0.68 . Employing a threshold of Probability (PeptideProphet) above 0.8 , we make sure the percent of false S-nitrosated peptides identified in this experiment is below $3.6 \%$. In this state, the fraction of all correct assignments passing MPT filter is nearly $75 \%$ (sensitivity). (B) Identification of the peptide AGCQVVPSDMMDGR in aminolevulinate delta dehydratase. Red dots indicate matching of $b$-ions, and blue dots indicate $y$-ions. Orange dots labeled with $b *$ indicate dehydrated $b$ ions. The purple dot at m/z 294.9 shows the ICAT diagnostic peak. (C) Reconstructed ion chromatogram of the precursor ion from peptide AGCQVVPSDMMDGR in its light and heavy versions using ASAPRatio. The ratio of light (C57/BL6) versus heavy (KK-Ay) ICAT labeled peptides was determined to be $0.46 \pm 0.03$. 


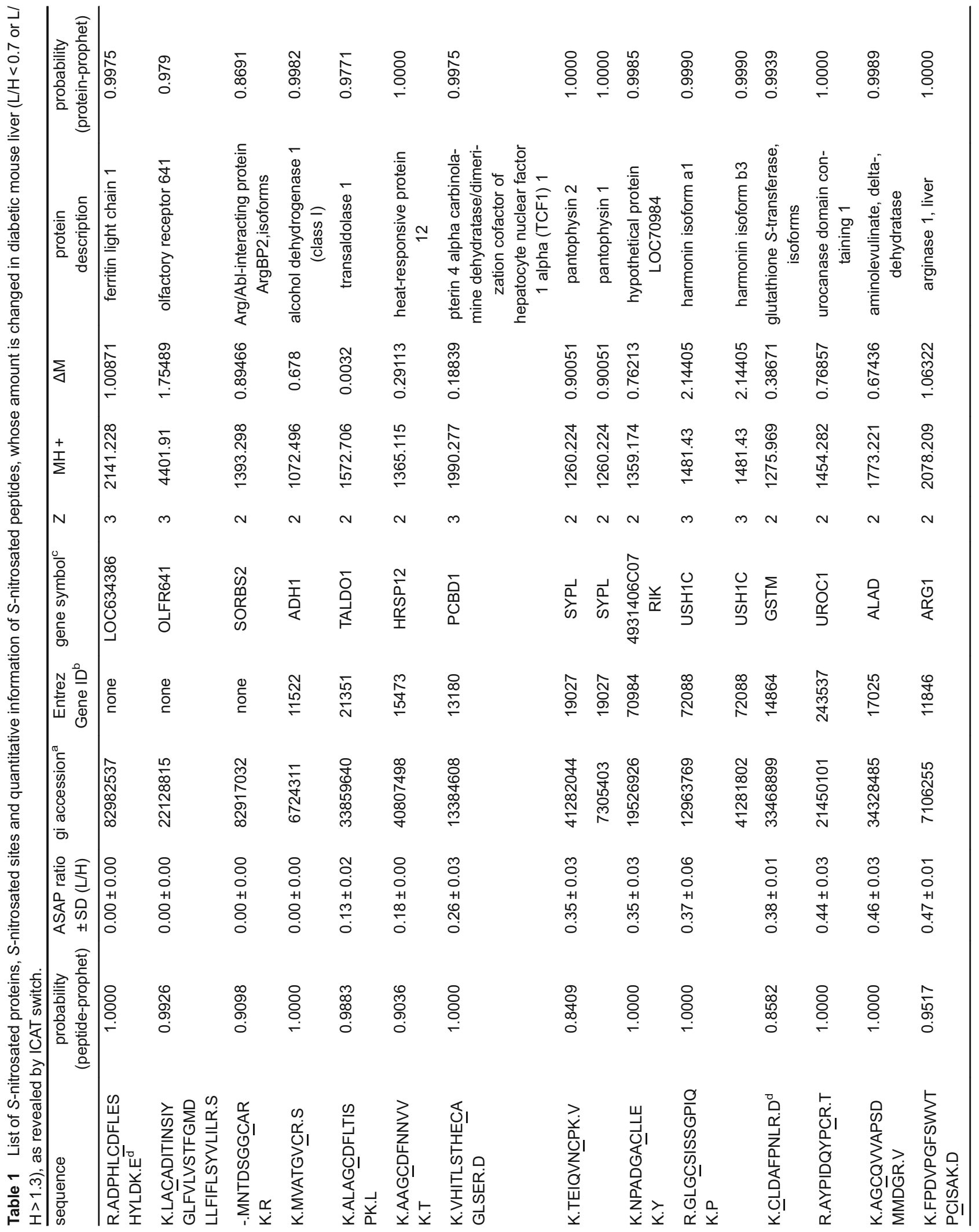




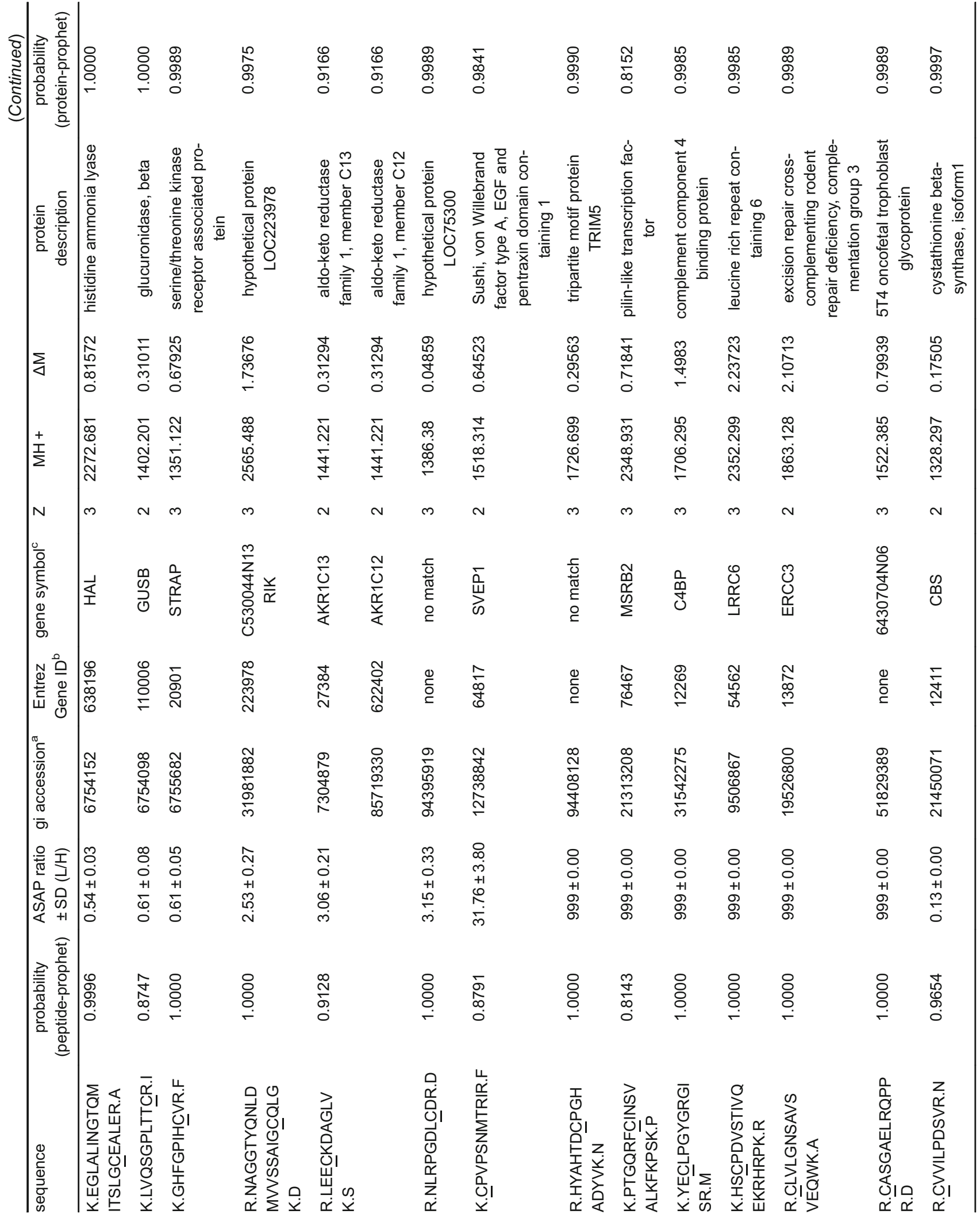




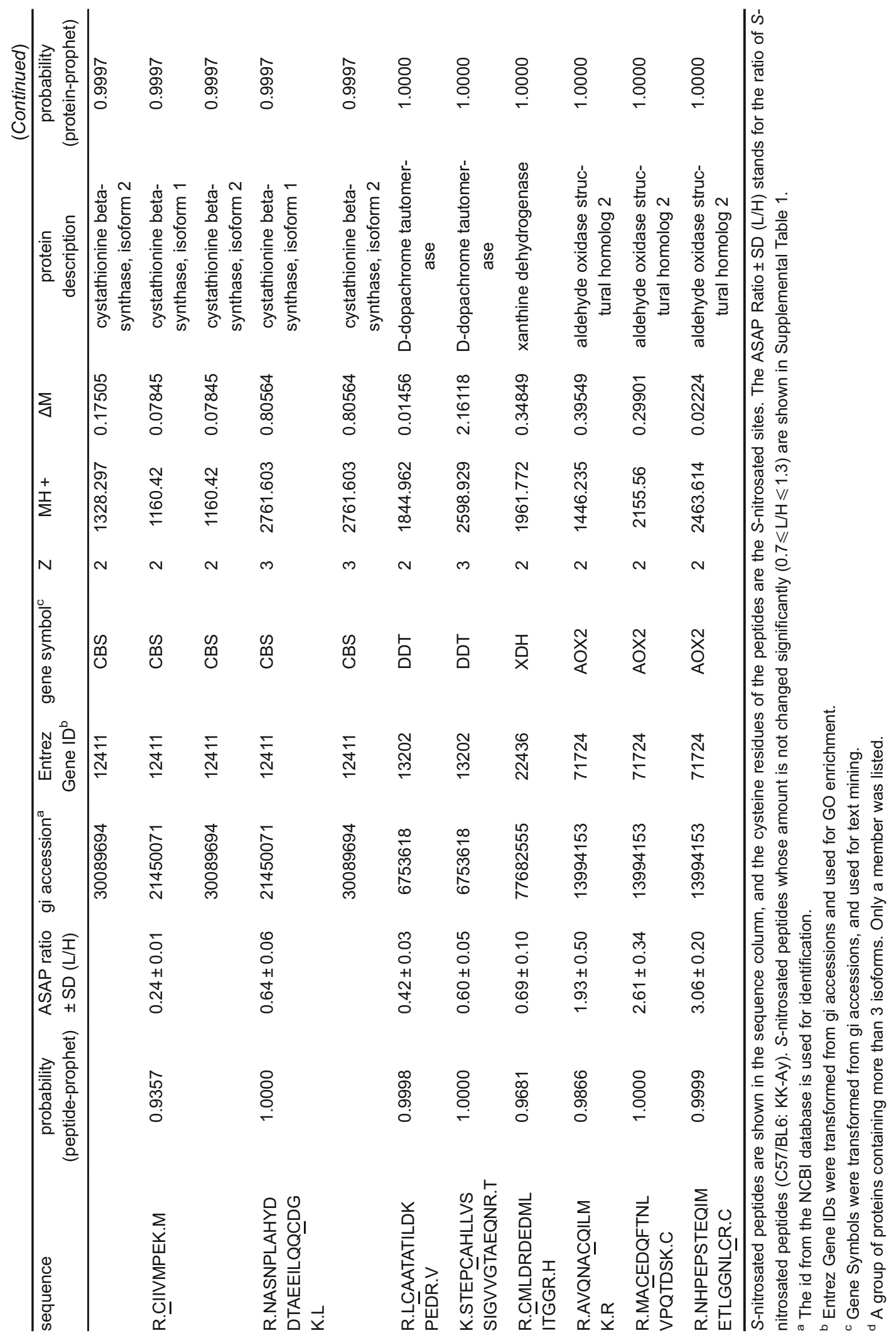


A

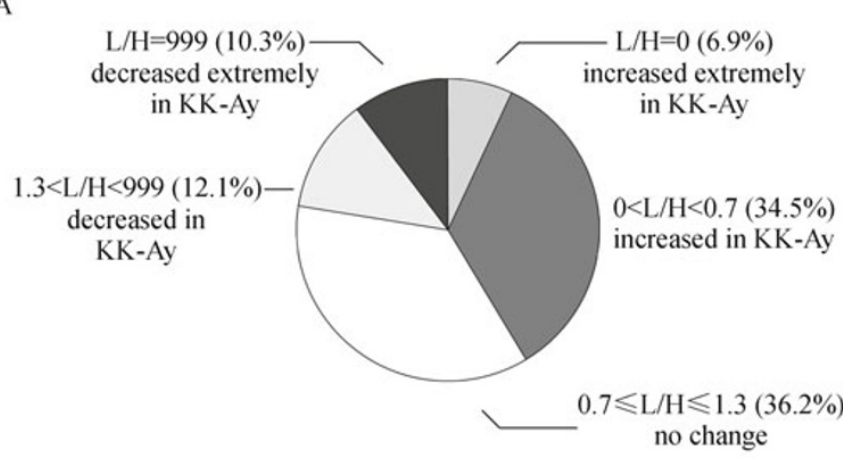

B

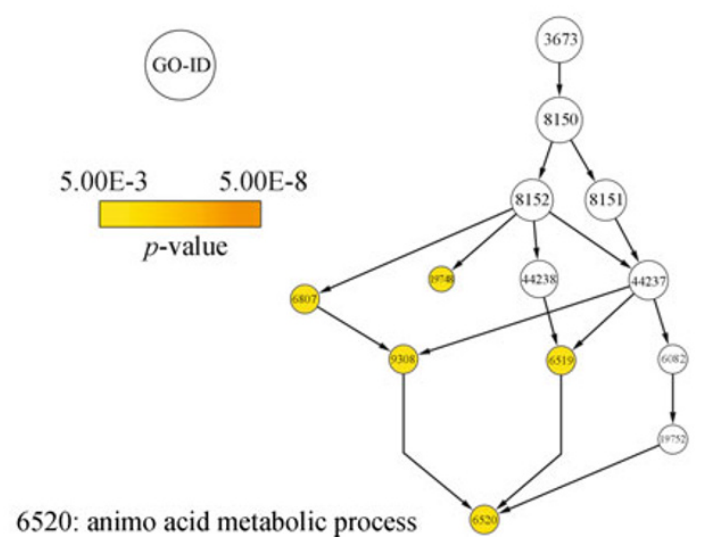

Figure 3. Analysis of S-nitrosated peptides and proteins identified in the normal and diabetic mouse liver using ICAT switch. (A) The number of $S$-nitrosated peptides distributed in different L/H (C57/BL6 versus KK-Ay) ratio groups. L/H $=0: S$ nitrosated peptide only presents in KK-Ay mouse liver; $0<\mathrm{L} / \mathrm{H}<0.7$ : $S$-nitrosation increased in KK-Ay mouse liver; $0.7 \leqslant \mathrm{~L} / \mathrm{H} \leqslant 1.3$ : no change in S-nitrosated peptides from KK-Ay mouse liver; $1.3<\mathrm{L} / \mathrm{H}<999$ : $\mathrm{S}$-nitrosation decreased in KK-Ay mouse liver; $\mathrm{L} / \mathrm{H}=999$ : $S$-nitrosated peptides only present in C57BL/6J mouse liver. (B) GO enrichment result of proteins with changed S-nitrosation level. Numbers in the nodes represented IDs of GO terms. The color from yellow to red (dependent on $p$-value) reflected their degree of enrichment.

aspartate aminotransferase (Lefièvre et al., 2007), and glutathione peroxidase 1 (Koh et al., 2001; Lindermayr et al., 2005), were also detected in our study, indicating the efficiency of the ICAT switch.

With this quantitative proteomic method, we were able to find a number of new $S$-nitrosated targets that are known to be involved in diabetes and thus merit special investigation. (A) Arg/Abl binding protein (ArgBP2), this multi-adaptor protein interacts with many important proteins in cell signaling (Wang et al., 1997), including Akt (Yuan et al., 2005) and cbl (Soubeyran et al., 2003), which are involved in the insulininduced glucose transport pathway. S-nitrosated ArgBP2 was only detected in KK-Ay mouse liver, suggesting that $S$ nitrosation of this protein may contribute to dysfunction of the glucose transport pathway. (B) Hepatocyte pterin 4 alpha carbinolamine dehydratase/dimerization cofactor (PCBD1), also known as dimerizing cofactor for HNF1 (DCoH), can bind to the HNF1 family of transcriptional activators, nuclear factor 1 alpha (TCF1) 1 (Mendel et al., 1991). The HNF1-DCoH complex regulates the expression of a large group of genes related to metabolism and the insulin pathway in liver. Defects in the expression of these genes underlie maturity-onset diabetes of the young (MODY) (Yamagata et al., 1996a, b), and mice lacking $\mathrm{DCoH}$ display hyperphenylalaninemia and an impaired glucose tolerance (Bayle et al., 2002). The His80 and Glu81 residues (Rose et al., 2004) are thought to be the active site of $\mathrm{DCoH} 1$, and the 3.9-fold increase in $S$ nitrosation of Cys82 in diabetic mouse liver detected in our experiment may affect the structure near the active site and cause dysfunction. (C) Pantophysin (SYPL) (Leube, 1994), it is reported to associate with GLUT4-containing vesicles and may regulate vesicle transport in adipocytes (Brooks et al., 2000). Here we found that $S$-nitrosation of this protein increased 2.9 folds in diabetic mouse liver. Whether this $S$ nitrosation affects Pantophysin's role in GLUT translocation is expected for further research. (D) Aminolevulinate delta dehydratase (ALAD), its activity decreased in the liver of high glucose-induced diabetic rats (Folmer et al., 2002) and the oxidation of cysteines is thought to be one possible reason (Afonso et al., 1996). Here we found that $S$-nitrosation of ALAD increased 2.17 folds in diabetic mouse liver, suggesting one possible contribution to the decrease of 


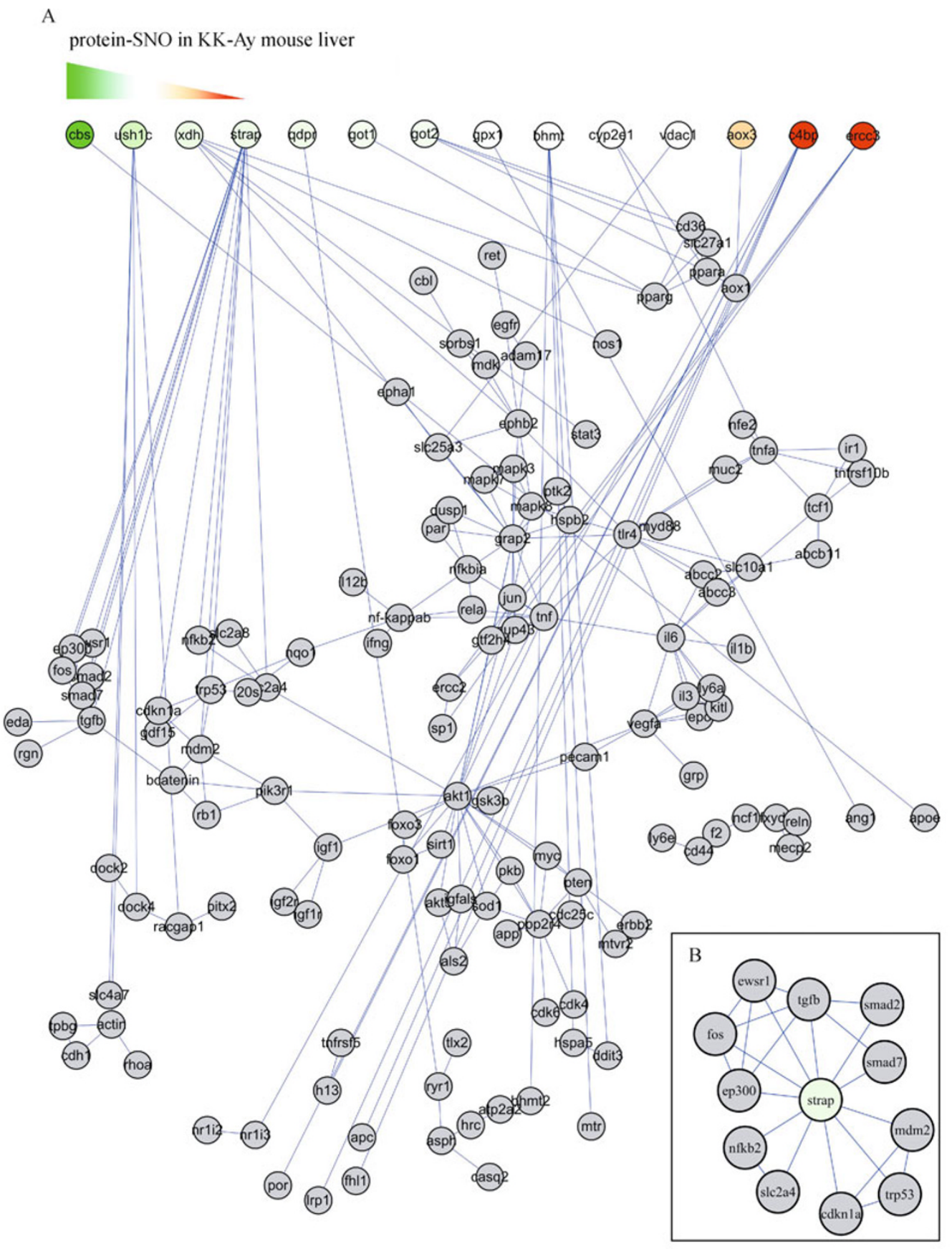

Figure 4. Integrative analysis of the network of S-nitrosated proteins. (A) Text-mining network constructed by S-nitrosated proteins. The nodes colored green, white or red stand for $S$-nitrosated proteins, and the gradient from green to red reflects the increasing ratio of light (C57/BL6) versus heavy (KK-Ay) ICAT labeled peptides. Grey nodes are proteins that affect or are affected by $S$-nitrosated proteins. (B) Topological connections of the S-nitrosated protein STRAP. Grey nodes stand for proteins related to STRAP by text-mining. The edge between two nodes stands for the relationship. Cdkn1a and trp53 are known to be regulated by STRAP from the information provided by this subnet, and these two proteins are associated with diabetes.

ALAD activity. (E) Aldo-keto reductase family 1, member C12/ C13 (AKR1C12/C13) (S-nitrosation decreased by 67\%), this superfamily is related to the pathogenesis of secondary complications associated with diabetes (Bhatnagar and
Srivastava, 1992). Aldose reductase (AR) is another member of the aldo-keto reductase superfamily, and the inhibition of $A R$ is thought to prevent diabetic complications. AR is very sensitive to nitric oxide donors and increasing NO 
availability can usefully inhibit the polyol pathway via downregulating $A R$ activity and alleviating the development of diabetes complications (Dixit et al., 2001; Chandra et al., 2002). The functional role of the decrease in the extent of $S$ nitrosation of AKR1C12/C13 might be one possible factor for the development of diabetes. (F) Alcohol dehydrogenase 1 (class I) (gi6724311), which was S-nitrosated only in KK-Ay mouse liver, could depress the phosphorylation of insulin receptor (Tyr1146) and reduced phosphorylation of Akt and GSK-3beta in cardiomyocytes from ethanol-fed mice ( $\mathrm{Li}$ and Ren, 2008). These are similar to the abnormal insulin signaling pathway in liver or peripheral diabetic tissues (Cohen, 2006).

In addition, with the help of GO enrichment, the integrative analysis results showed that the $S$-nitrosated proteins in KKAy mouse liver were enriched in processes related with amino acid metabolism (Fig. 3B). For example, one S-nitrosated target is arginase 1 (Entrez Gene ID: 11846), which is responsible for hydrolyzing L-arginine to form L-ornithine and urea, is involved in the nitrogen metabolism pathway and urea cycle. There are also reports that amino acid metabolism was abnormal in diabetes (Wijekoon et al., 2004; Noguchi et al., 2006). Excess amino acids in the liver can act as substrates for gluconeogenesis (Felig,1975), interfere with the inhibitory effect of insulin on glucose production (Boden and Tappy, 1990), and change the insulin/glucagon ratio (Roden et al., 1996), all of which may increase the output of hepatic glucose. Moreover, abnormal amino acid metabolism in the liver may change levels of amino acids throughout the whole body. Some reports suggest that high levels of amino acids can have both positive (Tremblay and Marette, 2001; Nobukuni et al., 2005) and negative (Armstrong et al., 2001; Bogan et al., 2001) effects on glucose metabolism and insulin action in peripheral tissues. Amino acid levels can also affect insulin secretion in pancreatic islet $\beta$-cells (Newsholme et al., 2007). Therefore, it is possible that $S$-nitrosation interferes with amino acid metabolic processes and thus contributes to diabetes.

Meanwhile, we noticed that not every S-nitrosated protein identified in our research can be linked with diabetes or its complications directly. Thus, we used text-mining network to help to reveal the relationships. As shown in Fig. 4B, this subnet composed by STRAP and nodes connected to it, such as p21 and p53. p21 is known to promote adipose tissue expansion and leads to insulin resistance (Inoue et al., 2008), and many proteins affecting the insulin pathway are regulated by p53 (Feng et al., 2007). This subnet indicates that $S$ nitrosation of STRAP may play an indirect role in diabetes via p21 and p53. Therefore, the network constructed for $S$ nitrosated proteins provided clues about the relationship between $S$-nitrosation and type 2 diabetes.

With the new developed ICAT switch method, we got the quantitative proteomic profile of S-nitrosation in C57/BL6 and KK-Ay mouse liver. The quantitative information, together with the network analysis of S-nitrosated targets contributed to explaining the relationship between $S$-nitrosation and diabetes. Our work provides a solution for quantification of multiple $S$-nitrosation, makes it possible to elucidate the integrative correlation of $S$-nitrosation with pathophysiologic processes. The mode of this work can be used to study $S$ nitrosation profiles in other physiologic and pathological processes.

\section{MATERIALS AND METHODS}

\section{Reagents}

Cleavable ICAT $^{\mathrm{TM}}$ reagent kits were purchased from Applied Biosystems (Foster City, CA, USA), Methyl methanethiosulfonate (MMTS), biotin-(N-(6-(Biotinamido)hexyl)-3'-(2'-pyridyldithio)-propionamide (biotin-HPDP), and $\mathrm{BCA}^{\mathrm{TM}}$ protein assay kits were from Pierce (Rockford, IL, USA). PlusOne ${ }^{\mathrm{TM}}$ urea was from GE Healthcare (Piscataway, NJ, USA). Protease inhibitor cocktail tablets (Complete, Mini, EDTA-free) were from Roche Applied Sciences (Indianapolis, IN, USA). Sequencing-grade modified trypsin (V5113) was from Promega (Madison, WI, USA). Solvents used in LC/MS analysis, including formic acid (FA), acetonitrile ( $A C N$ ) and methanol, were from J.T. Baker Chemicals (Philipsburg, NJ, USA). All other chemicals were from Sigma-Aldrich (St. Louis, MO, USA).

\section{Animals}

Eight-week-old female lean wild-type C57BL/6J and KK-Ay mice were obtained from the Institute of Experimental Animals, Beijing (Beijing, China). The mice were housed in mesh cages in a room maintained at $25^{\circ} \mathrm{C}$ and illuminated in $12: 12-\mathrm{h}$ light/dark cycles. They were provided with a standard rodent diet and water. All animal experimental procedures were conducted according to the Animal Welfare Act 2006.

\section{Protein Preparation}

Livers from C57BL/6J and KK-Ay mice were homogenized separately in detergent-free lysis buffer $(50 \mathrm{mM}$ Tris, $\mathrm{pH}$ 7.7, 2.5 mM EDTA, $0.1 \mathrm{mM}$ neocuproine, $8 \mathrm{M}$ Urea, $20 \mathrm{mM}$ MMTS and protease inhibitor cocktail) on ice, as described in the literature (Han and Chen, 2008). Lysates were centrifuged at $12,000 \mathrm{~g}$ for $20 \mathrm{~min}$ at $4^{\circ} \mathrm{C}$ to remove the debris. All steps were performed in the dark. Protein concentration was determined using a $\mathrm{BCA}^{\mathrm{TM}}$ protein assay kit and adjusted to $1 \mathrm{mg} / \mathrm{mL}$, after removal of MMTS from the protein solution by TCA/ acetone precipitation.

In each individual experiment, one control mouse and one diabetic KK-ay mouse were used. Independent experiments were conducted for 9 times and representative data were presented.

\section{ICAT switch method for isolation of S-nitrosated peptides}

Protein samples were dissolved in blocking buffer $(20 \mathrm{mM}$ Tris, $\mathrm{pH}$ 7.7, $1 \mathrm{mM}$ EDTA, $0.1 \mathrm{mM}$ neocuproine, $8 \mathrm{M}$ urea, $20 \mathrm{mM}$ MMTS) and incubated for $1 \mathrm{~h}$ at room temperature in dark (Han and Chen, 2008). After TCA/acetone precipitation, the pellets were dissolved in 
labeling buffer (20 mM Tris, pH 7.7, 1 mM EDTA, 0.1 mM neocuproine, $4 \mathrm{M}$ urea and a vial of ICAT reagents) in the presence of sodium ascorbate. Protein obtained from C57BL/6J and KK-Ay mice was labeled with isotopically light and heavy ICAT reagents, respectively, and incubated for $2 \mathrm{~h}$ at room temperature. Labeled preparations were combined and precipitated by TCA/acetone. Pellets were dissolved in enzymolysis buffer $\left(50 \mathrm{mM} \mathrm{NH}_{4} \mathrm{HCO}_{3}, \mathrm{pH} 8.3,1.5 \mathrm{M}\right.$ urea) and digested with trypsin overnight at $37^{\circ} \mathrm{C}$ using an enzyme-toprotein ratio of 1:50. The resulting peptide mixture was cleaned using a cation-exchange cartridge. Cleaned peptides were collected and subjected to affinity purification using monomeric avidin agarose. Peptides labeled with ICAT reagents were enriched and then cleaved with cleaving reagent at $37^{\circ} \mathrm{C}$ for $2 \mathrm{~h}$. The cation-exchange cartridge, cleaving reagent and relevant buffers were from a cleavable ICAT ${ }^{\mathrm{TM}}$ reagents kit, and procedures for purifying peptides were according to the manufacturer's guidelines. ICAT labeled peptides were dried by vacuum centrifuge and resolubilized in $0.1 \%$ formic acid for LC-MS/MS analysis.

\section{LC/MS/MS analysis}

Peptides from which the biotin tag had been removed were separated by reverse-phase capillary liquid chromatography (RP-C18, Column Technology Inc, $0.15 \mathrm{~mm} \times 150 \mathrm{~mm}$ ) at a flow rate of $200 \mathrm{~nL} / \mathrm{min}$. The eluent was directly analyzed by a Thermo LTQ linear trap instrument equipped with a Thermo micro-electrospray source, a Thermo Surveyor pump and an autosampler (Thermo Finnigan, San Jose, CA, USA). A survey scan followed by 5 CID (collision induced dissociation) events was used. Peptide identification by CID was carried out in automated mode using the 3-min dynamic exclusion option.

\section{Database search, data validation and quantification}

MS/MS spectra were searched against a database downloaded from the National Center for Biotechnology Information website (NCBI; mouse; May 4, 2007; 46,903 entries) using SEQUEST (Yates et al., 1995) in Bioworks 3.2 (Thermo Finnigan). The SEQUEST database search criteria included a static modification of cysteine residues of $227 \mathrm{Da}$ (light cleavable ICAT reagent) and a variable modification of $9 \mathrm{Da}$ for cysteines (for the heavy cleavable ICAT reagent). The tolerance of the precursor ion and fragment ion were 2 and $1 \mathrm{amu}$, respectively. Peptides were permitted to have up to two missed cleavages. The .dta and .out files obtained from SEQUEST were validated using PeptideProphet (Keller et al., 2002) and ProteinProphet (Nesvizhskii et al., 2003). The minimal probability thresholds (MPT) were set to make false-positive error rates for both peptides and proteins were below 0.05 . The peptides passing the MPT statistic filter in corresponding experiment were accepted and quantified. The minimal intensity of precursor ions peak was set to 1000 when acquiring MS/MS data. Relative quantification with SIC (single ion chromatogram) was performed using ASAPRatio (Li et al., 2003). ASAPRatio grouped abundance ratios of same sequence peptide (including different isotopic forms, charge states, repeats) in one RP elution peak together to calculate the mean and standard deviation first, then abundance ratios of same sequence peptide in different RP elution peaks were grouped together to get the final mean and standard deviation for a unique peptide. Software packages were utilized via the Trans-Proteomics Pipeline (ISB, Seattle, WA, USA).
The resulting peptide spectra of proteins were manually checked for qualitative and quantitative results.

\section{Bioinformatic analysis of the results of $S$-nitrosation}

The database ID (gi Accession) of the S-nitrosated proteins was converted into an Entrez Gene ID using the DAVID Gene ID Conversion Tool (Dennis et al., 2003). Entrez Gene IDs were used to do GO enrichment. S-nitrosated proteins were visualized with Cytoscape (Shannon et al., 2003) and used to do GO enrichment using the BiNGO (Maere et al., 2005) plugin with the Gene Ontology (GO) annotation (Ashburner et al., 2000). The Benjamini and Hochberg False Discovery Rate (FDR) correction was chosen as the testing correction, and a $p$-value threshold of 0.05 was selected for Biological Process categories in the BiNGO settings. A text-mining network was constructed for $S$-nitrosated proteins and their related proteins via the Agilent Literature Search (Vailaya et al., 2005) plugin of Cytoscape in order to analyze the contribution of the S-nitrosated proteins to diabetes through their impact on other proteins. In the Agilent Literature Search tool, Gene Symbols of S-nitrosated proteins were used to search the Pubmed database; "Mus musculus" was set as the concept lexicon; Max Engine Matches was set to 10; and aliases were used. The network was displayed by Cytoscape, and relationships between nodes were checked manually. Wrong nodes and edges were removed, and nodes standing for S-nitrosated proteins were colored using a green-to-red gradient according to the amount of $S$-nitrosated peptides.

\section{ACKNOWLEDGEMENTS}

We are grateful to Prof. Rong Zeng, Prof. Taijiao Jiang and Prof. John Yates III for valuable suggestions and discussions. This research was supported by the National Basic Research Program (973 program) of China (Grant Nos. 2006CB911001, 2005CB522804), the National Natural Science Foundation of China (Grant Nos. 90606020 and 30770512) and the Knowledge Innovation Program of the Chinese Academy of Sciences.

\section{ABBREVIATIONS}

biotin-HPDP, biotin-(N-(6-(biotinamido)hexyl)-3'-(2'-pyridyldithio)-propionamide; CID, collision induced dissociation; DAG, directed acyclic graph; GLUT, glucose transporter; GO, gene ontology; GSNO, Snitrosoglutathione; ICAT, isotope-coded affinity tag; iNOS, inducible NO synthase; MMTS, methyl methane thiosulfonate; MODY, maturity onset diabetes of the young; MPT, minimal probability threshold; NO, nitric oxide; RNS, Reactive nitrogen species; SNOSID, SNO-site identification; SNO, S-nitrosothiol; STRAP, serine/threonine kinase receptor-associated protein; T2DM, type 2 diabetes mellitus

\section{REFERENCES}

Afonso, S.G., Polo, C.F., Enriquez de Salamanca, R., and Batlle, A. (1996). Mechanistic studies on uroporphyrin I-induced photoinactivation of some heme-enzymes. Int J Biochem Cell Biol 28, 415-420.

Armstrong, J.L., Bonavaud, S.M., Toole, B.J., and Yeaman, S.J. (2001). Regulation of glycogen synthesis by amino acids in cultured human muscle cells. J Biol Chem 276, 952-956. 
Ashburner, M., Ball, C.A., Blake, J.A., Botstein, D., Butler, H., Cherry, J.M., Davis, A.P., Dolinski, K., Dwight, S.S., Eppig, J.T., et al. (2000). Gene ontology: tool for the unification of biology. The Gene Ontology Consortium. Nat Genet 25, 25-29.

Bayle, J.H., Randazzo, F., Johnen, G., Kaufman, S., Nagy, A., Rossant, J., and Crabtree, G.R. (2002). Hyperphenylalaninemia and impaired glucose tolerance in mice lacking the bifunctional DCoH gene. J Biol Chem 277, 28884-28891.

Benhar, M., and Stamler, J.S. (2005). A central role for S-nitrosylation in apoptosis. Nat Cell Biol 7, 645-646.

Bhatnagar, A., and Srivastava, S.K. (1992). Aldose reductase: congenial and injurious profiles of an enigmatic enzyme. Biochem Med Metab Biol 48, 91-121.

Boden, G., and Tappy, L. (1990). Effects of amino acids on glucose disposal. Diabetes 39, 1079-1084

Bogan, J.S., McKee, A.E., and Lodish, H.F. (2001). Insulinresponsive compartments containing GLUT4 in 3T3-L1 and CHO cells: regulation by amino acid concentrations. Mol Cell Biol 21, 4785-4806.

Brooks, C.C., Scherer, P.E., Cleveland, K., Whittemore, J.L., Lodish, H.F., and Cheatham, B. (2000). Pantophysin is a phosphoprotein component of adipocyte transport vesicles and associates with GLUT4-containing vesicles. J Biol Chem 275, 2029-2036.

Carvalho-Filho, M.A., Ueno, M., Hirabara, S.M., Seabra, A.B., Carvalheira, J.B., de Oliveira, M.G., Velloso, L.A., Curi, R., and Saad, M.J. (2005). S-nitrosation of the insulin receptor, insulin receptor substrate 1 , and protein kinase B/Akt: a novel mechanism of insulin resistance. Diabetes 54, 959-967.

Ceriello, A., Quagliaro, L., D’Amico, M., Di Filippo, C., Marfella, R., Nappo, F., Berrino, L., Rossi, F., and Giugliano, D. (2002). Acute hyperglycemia induces nitrotyrosine formation and apoptosis in perfused heart from rat. Diabetes 51, 1076-1082.

Chandra, D., Jackson, E.B., Ramana, K.V., Kelley, R., Srivastava, S. K., and Bhatnagar, A. (2002). Nitric oxide prevents aldose reductase activation and sorbitol accumulation during diabetes. Diabetes 51, 3095-3101.

Cohen, P. (2006). The twentieth century struggle to decipher insulin signalling. Nat Rev Mol Cell Biol 7, 867-873.

Dennis, G. Jr, Sherman, B.T., Hosack, D.A., Yang, J., Gao, W., Lane, H.C., and Lempicki, R.A. (2003). DAVID: Database for Annotation, Visualization, and Integrated Discovery. Genome Biol 4, 3-13.

Dixit, B. L., Ramana, K. V., Chandra, D., Jackson, E. B., Srivastava, S., Bhatnagar, A., and Srivastava, S. K. (2001). Metabolic regulation of aldose reductase activity by nitric oxide donors. Chem Biol Interact 130-132, 573-581.

Felig, P. (1975). Amino acid metabolism in man. Annu Rev Biochem 44, 933-955.

Feng, Z., Hu, W., de Stanchina, E., Teresky, A.K., Jin, S., Lowe, S., and Levine, A.J. (2007). The regulation of AMPK beta1, TSC2, and PTEN expression by p53: stress, cell and tissue specificity, and the role of these gene products in modulating the IGF-1-AKT-mTOR pathways. Cancer Res 67, 3043-3053.

Folmer, V., Soares, J.C., and Rocha, J.B. (2002). Oxidative stress in mice is dependent on the free glucose content of the diet. Int $\mathrm{J}$ Biochem Cell Biol 34, 1279-1285.

Fujimoto, M., Shimizu, N., Kunii, K., Martyn, J.A., Ueki, K., and Kaneki, M. (2005). A role for iNOS in fasting hyperglycemia and impaired insulin signaling in the liver of obese diabetic mice.
Diabetes 54, 1340-1348.

Gygi, S.P., Rist, B., Gerber, S.A., Turecek, F., Gelb, M.H., and Aebersold, R. (1999). Quantitative analysis of complex protein mixtures using isotope-coded affinity tags. Nat Biotechnol 17, 994-999.

Halder, S.K., Anumanthan, G., Maddula, R., Mann, J., Chytil, A., Gonzalez, A.L., Washington, M.K., Moses, H.L., Beauchamp, R. D., and Datta, P.K. (2006). Oncogenic function of a novel WDdomain protein, STRAP, in human carcinogenesis. Cancer Res 66 , 6156-6166.

Han, P., and Chen, C. (2008). Detergent-free biotin switch combined with liquid chromatography/tandem mass spectrometry in the analysis of S-nitrosylated proteins. Rapid Commun Mass Spectrom 22, 1137-1145.

Hao, G., Derakhshan, B., Shi, L., Campagne, F., and Gross, S.S. (2006). SNOSID, a proteomic method for identification of cysteine S-nitrosylation sites in complex protein mixtures. Proc Natl Acad Sci U S A 103, 1012-1017.

Hess, D.T., Matsumoto, A., Kim, S.O., Marshall, H.E., and Stamler, J. S. (2005). Protein S-nitrosylation: purview and parameters. Nat Rev Mol Cell Biol 6, 150-166.

Inoue, N., Yahagi, N., Yamamoto, T., Ishikawa, M., Watanabe, K., Matsuzaka, T., Nakagawa, Y., Takeuchi, Y., Kobayashi, K., Takahashi, A., et al. (2008). Cyclin-dependent kinase inhibitor, p21WAF1/CIP1, is involved in adipocyte differentiation and hypertrophy, linking to obesity and insulin resistance. J Biol Chem 283, 21220-21229.

Jaffrey, S.R., and Snyder, S.H. (2001). The biotin switch method for the detection of S-nitrosylated proteins. Sci. STKE 2001, L1.

Jung, H., Seong, H. A., and Ha, H. (2007). NM23-H1 tumor suppressor and its interacting partner STRAP activate p53 function. J Biol Chem 282, 35293-35307.

Keller, A., Nesvizhskii, A.I., Kolker, E., and Aebersold, R. (2002). Empirical statistical model to estimate the accuracy of peptide identifications made by MS/MS and database search. Anal Chem 74, 5383-5392.

Koh, Y.H., Suzuki, K., Che, W., Park, Y.S., Miyamoto, Y., Higashiyama, S., and Taniguchi, N. (2001). Inactivation of glutathione peroxidase by $\mathrm{NO}$ leads to the accumulation of $\mathrm{H} 2 \mathrm{O} 2$ and the induction of HB-EGF via C-Jun NH2-terminal kinase in rat aortic smooth muscle cells. FASEB J 15, 1472-1474.

Lefièvre, L., Chen, Y., Conner, S.J., Scott, J.L., Publicover, S.J., Ford, W.C., and Barratt, C.L. (2007). Human spermatozoa contain multiple targets for protein S-nitrosylation: an alternative mechanism of the modulation of sperm function by nitric oxide? Proteomics 7, 3066-3084.

Leube, R.E. (1994). Expression of the synaptophysin gene family is not restricted to neuronal and neuroendocrine differentiation in rat and human. Differentiation 56, 163-171.

Li, S.Y., and Ren, J. (2008). Cardiac overexpression of alcohol dehydrogenase exacerbates chronic ethanol ingestion-induced myocardial dysfunction and hypertrophy: role of insulin signaling and ER stress. J Mol Cell Cardiol 44, 992-1001.

Li, X.J., Zhang, H., Ranish, J.A., and Aebersold, R. (2003). Automated statistical analysis of protein abundance ratios from data generated by stable-isotope dilution and tandem mass spectrometry. Anal Chem 75, 6648-6657.

Lindermayr, C., Saalbach, G., and Durner, J. (2005). Proteomic 
identification of S-nitrosylated proteins in Arabidopsis. Plant Physiol 137, 921-930.

Maere, S., Heymans, K., and Kuiper, M. (2005). BiNGO: a Cytoscape plugin to assess overrepresentation of gene ontology categories in biological networks. Bioinformatics 21, 3448-3449.

Mendel, D.B., Khavari, P.A., Conley, P.B., Graves, M.K., Hansen, L.P., Admon, A., and Crabtree, G.R. (1991). Characterization of a cofactor that regulates dimerization of a mammalian homeodomain protein. Science 254, 1762-1767.

Nesvizhskii, A.I., Keller, A., Kolker, E., and Aebersold, R. (2003). A statistical model for identifying proteins by tandem mass spectrometry. Anal Chem 75, 4646-4658.

Newsholme, P., Bender, K., Kiely, A., and Brennan, L. (2007). Amino acid metabolism, insulin secretion and diabetes. Biochem Soc Trans 35, 1180-1186.

Nobukuni, T., Joaquin, M., Roccio, M., Dann, S.G., Kim, S.Y., Gulati, P., Byfield, M.P., Backer, J.M., Natt, F., Bos, J.L., et al. (2005). Amino acids mediate mTOR/raptor signaling through activation of class 3 phosphatidylinositol $30 \mathrm{H}$-kinase. Proc Natl Acad Sci U S A 102, 14238-14243.

Noguchi, Y., Zhang, Q.W., Sugimoto, T., Furuhata, Y., Sakai, R., Mori, M., Takahashi, M., and Kimura, T. (2006). Network analysis of plasma and tissue amino acids and the generation of an amino index for potential diagnostic use. Am J Clin Nutr 83, 513S-519S.

Perreault, M., and Marette, A. (2001). Targeted disruption of inducible nitric oxide synthase protects against obesity-linked insulin resistance in muscle. Nat Med 7, 1138-1143.

Qu, J., Liu, G.H., Wu, K., Han, P., Wang, P., Li, J., Zhang, X., and Chen, C. (2007). Nitric oxide destabilizes Pias3 and regulates sumoylation. PLoS ONE 2, e1085.

Roden, M., Perseghin, G., Petersen, K. F., Hwang, J. H., Cline, G. W., Gerow, K., Rothman, D. L., and Shulman, G. I. (1996). The roles of insulin and glucagon in the regulation of hepatic glycogen synthesis and turnover in humans. J Clin Invest 97, 642-648.

Rose, R.B., Pullen, K.E., Bayle, J.H., Crabtree, G.R., and Alber, T. (2004). Biochemical and structural basis for partially redundant enzymatic and transcriptional functions of $\mathrm{DCoH}$ and $\mathrm{DCoH} 2$. Biochemistry 43, 7345-7355.

Shannon, P., Markiel, A., Ozier, O., Baliga, N.S., Wang, J.T., Ramage, D., Amin, N., Schwikowski, B., and Ideker, T. (2003). Cytoscape: a software environment for integrated models of biomolecular interaction networks. Genome Res 13, 2498-2504.

Sies, H., Dafré, A.L., Ji, Y., and Akerboom, T.P. (1998). Protein Sthiolation and redox regulation of membrane-bound glutathione transferase. Chem Biol Interact 111-112, 177-185.

Soubeyran, P., Barac, A., Szymkiewicz, I., and Dikic, I. (2003). CblArgBP2 complex mediates ubiquitination and degradation of c-Abl. Biochem J 370, 29-34.

Sugita, H., Kaneki, M., Tokunaga, E., Sugita, M., Koike, C., Yasuhara,
S., Tompkins, R.G., and Martyn, J.A. (2002). Inducible nitric oxide synthase plays a role in LPS-induced hyperglycemia and insulin resistance. Am J Physiol Endocrinol Metab 282, E386-E394.

Tremblay, F., and Marette, A. (2001). Amino acid and insulin signaling via the mTOR/p70 S6 kinase pathway. A negative feedback mechanism leading to insulin resistance in skeletal muscle cells. J Biol Chem 276, 38052-38060.

Vailaya, A., Bluvas, P., Kincaid, R., Kuchinsky, A., Creech, M., and Adler, A. (2005). An architecture for biological information extraction and representation. Bioinformatics 21, 430-438.

Wadham, C., Parker, A., Wang, L., and Xia, P. (2007). High glucose attenuates protein S-nitrosylation in endothelial cells: role of oxidative stress. Diabetes 56, 2715-2721.

Wang, B., Golemis, E.A., and Kruh, G.D. (1997). ArgBP2, a multiple Src homology 3 domain-containing, Arg/Abl-interacting protein, is phosphorylated in v-Abl-transformed cells and localized in stress fibers and cardiocyte Z-disks. J Biol Chem 272, 17542-17550.

Wellen, K.E., and Hotamisligil, G.S. (2005). Inflammation, stress, and diabetes. J Clin Invest 115, 1111-1119.

Wijekoon, E.P., Skinner, C., Brosnan, M.E., and Brosnan, J.T. (2004). Amino acid metabolism in the Zucker diabetic fatty rat: effects of insulin resistance and of type 2 diabetes. Can J Physiol Pharmacol 82, 506-514.

Xie, Q.W., Kashiwabara, Y., and Nathan, C. (1994). Role of transcription factor NF-kappa B/Rel in induction of nitric oxide synthase. J Biol Chem 269, 4705-4708.

Yamagata, K., Furuta, H., Oda, N., Kaisaki, P.J., Menzel, S., Cox, N. J., Fajans, S.S., Signorini, S., Stoffel, M., and Bell, G.I. (1996a). Mutations in the hepatocyte nuclear factor-4alpha gene in maturityonset diabetes of the young (MODY1). Nature 384, 458-460.

Yamagata, K., Oda, N., Kaisaki, P.J., Menzel, S., Furuta, H., Vaxillaire, M., Southam, L., Cox, R.D., Lathrop, G.M., Boriraj, V.V., et al. (1996b). Mutations in the hepatocyte nuclear factor-1alpha gene in maturity-onset diabetes of the young (MODY3). Nature 384, $455-458$.

Yasukawa, T., Tokunaga, E., Ota, H., Sugita, H., Martyn, J.A., and Kaneki, M. (2005). S-nitrosylation-dependent inactivation of Akt/ protein kinase B in insulin resistance. J Biol Chem 280, 7511-7518.

Yates, J.R. 3rd, Eng, J.K., McCormack, A.L., and Schieltz, D. (1995). Method to correlate tandem mass spectra of modified peptides to amino acid sequences in the protein database. Anal Chem 67, 1426-1436.

Yuan, Z.Q., Kim, D., Kaneko, S., Sussman, M., Bokoch, G.M., Kruh, G.D., Nicosia, S.V., Testa, J.R., and Cheng, J.Q. (2005). ArgBP2gamma interacts with Akt and p21-activated kinase-1 and promotes cell survival. J Biol Chem 280, 21483-21490.

Zimmet, P. (2000). Globalization, coca-colonization and the chronic disease epidemic: can the Doomsday scenario be averted? J Intern Med 247, 301-310. 